\title{
An NTCP Analysis of Urethral Complications from Low Doserate Mono- and Bi-Radionuclide Brachytherapy
}

\author{
V. E. Nuttens, ${ }^{1}$ A. E. Nahum, ${ }^{2}$ and S. Lucas ${ }^{1}$ \\ ${ }^{1}$ NAmur Research Institute for LIfe Sciences (NARILIS), Research Center for the Physics of Matter and Radiation (PMR-LARN), \\ University of Namur (FUNDP), Rue de Bruxelles, 61, 5000 Namur, Belgium \\ ${ }^{2}$ Department of Physics, Clatterbridge Center for Oncology, Clatterbridge Road Bebington, Merseyside CH63 4JY, UK
}

Correspondence should be addressed to S. Lucas, stephane.lucas@fundp.ac.be

Received 13 January 2011; Accepted 2 May 2011

Academic Editor: M. J. Zelefsky

Copyright () 2011 V. E. Nuttens et al. This is an open access article distributed under the Creative Commons Attribution License, which permits unrestricted use, distribution, and reproduction in any medium, provided the original work is properly cited.

\begin{abstract}
Urethral NTCP has been determined for three prostates implanted with seeds based on ${ }^{125} \mathrm{I}(145 \mathrm{~Gy}),{ }^{103} \mathrm{Pd}(125 \mathrm{~Gy})$, ${ }^{131} \mathrm{Cs}$ (115 Gy), ${ }^{103} \mathrm{Pd}-{ }^{125} \mathrm{I}$ (145 Gy), or ${ }^{103} \mathrm{Pd}-{ }^{131} \mathrm{Cs}$ (115 Gy or $130 \mathrm{~Gy}$ ). First, $\mathrm{DU}_{20}$, meaning that $20 \%$ of the urhral volume receive a dose of at least $\mathrm{DU}_{20}$, is converted into an I-125 LDR equivalent $\mathrm{DU}_{20}$ in order to use the urethral NTCP model. Second, the propagation of uncertainties through the steps in the NTCP calculation was assessed in order to identify the parameters responsible for large data uncertainties. Two sets of radiobiological parameters were studied. The NTCP results all fall in the 19\%-23\% range and are associated with large uncertainties, making the comparison difficult. Depending on the dataset chosen, the ranking of NTCP values among the six seed implants studied changes. Moreover, the large uncertainties on the fitting parameters of the urethral NTCP model result in large uncertainty on the NTCP value. In conclusion, the use of NTCP model for permanent brachytherapy is feasible but it is essential that the uncertainties on the parameters in the model be reduced.
\end{abstract}

\section{Introduction}

Radiotherapy treatment planning systems incorporating "biological" models are beginning to make their way into clinical use. The biological models in questions are for Tumor Control Probability (TCP) $[3,4]$ and Normal Tissue Complication Probability (NTCP) $[5,6]$. A common suggestion is that treatment plans be optimized to maximize the TCP while not exceeding a fixed, acceptable NTCP $[7,8]$. A considerable amount of work has been done to implement this biological model-based approach in permanent seed prostate brachytherapy [1,9-12]. Prostate brachytherapy morbidity is generally reported for the urethra [13-17] and rectum [1821]. Zaider et al. have extended radiotherapy NTCP models for these organs to Low Dose Rate (LDR) permanent brachytherapy $[1,12]$.

Three radionuclides are generally used in permanent prostate brachytherapy: iodine-125, palladium-103, and cesium-131. Each radionuclide has its advantages but also its drawbacks. ${ }^{125} \mathrm{I}$ and ${ }^{131} \mathrm{Cs}$ have similar emission spectrum whose mean energies are 28.37 and $30.45 \mathrm{keV}$, respectively.
${ }^{103} \mathrm{Pd}$ has a mean emitted energy of $20.74 \mathrm{keV}$ which reduces the dose to the surrounding organs at risk (OAR) such as rectum and urethra but also increases the risk of cold spots (underdosage) in the prostate. By contrast, the ${ }^{125} \mathrm{I}$ and ${ }^{131} \mathrm{Cs}$ dose distributions extend to larger distances, thus reducing the likelihood of cold spots at the cost of delivering more to the OARs for a given prostate dose. Urethral and rectal complications are reported at a similar frequency for ${ }^{125} \mathrm{I}$ or ${ }^{131}$ Cs [22].

The contrast between the properties of ${ }^{103} \mathrm{Pd},{ }^{125} \mathrm{I}$, and ${ }^{131} \mathrm{Cs}$ has motivated our research in developing a new kind of seed based on a mixture of two radionuclides, namely, ${ }^{103} \mathrm{Pd}_{0.75}{ }^{125} \mathrm{I}_{0.25}$ or ${ }^{103} \mathrm{Pd}_{0.25}{ }^{-131} \mathrm{Cs}_{0.75}$. The subscripts denote the fractions of internal activity of each radionuclide as defined in a previous paper [23]. To avoid a cumbersome style, the ${ }^{103} \mathrm{Pd}_{0.75}{ }^{-125} \mathrm{I}_{0.25}$ and ${ }^{103} \mathrm{Pd}_{0.25}{ }^{131} \mathrm{Cs}_{0.75}$ mixture will be referred to in the text as Pd-I and Pd-Cs. The dosimetry characteristics and prescription doses of these sources were derived in previous studies $[23,24]$.

In our study, we use the Zaider et al. NTCP model for the urethra to compare a monoradionuclide seed implant 
TABle 1: Prostate and urethra volumes for each planned patient.

\begin{tabular}{lccc}
\hline Patient & p01 & p02 & p03 \\
\hline Prostate volume (cc) & 24.64 & 38.9 & 45.33 \\
Urethra volume (cc) & 0.32 & 0.19 & 0.28 \\
\hline
\end{tabular}

TABle 2: Prescription dose for the different seeds used for planning.

\begin{tabular}{lccccc}
\hline & ${ }^{103} \mathrm{Pd}$ & ${ }^{125} \mathrm{I}$ & ${ }^{131} \mathrm{Cs}$ & $\mathrm{Pd}-\mathrm{I}$ & $\mathrm{Pd}-\mathrm{Cs}$ \\
\hline Internal activity & $100 \%$ & $100 \%$ & $100 \%$ & $75 \%-25 \%$ & $25 \%-75 \%$ \\
Prescription dose (Gy) & 125 & 145 & 115 & 145 & $\begin{array}{c}130(\mathrm{a}) \\
\end{array}$ \\
\hline
\end{tabular}

${ }^{103} \mathrm{Pd},{ }^{125} \mathrm{I}$ or ${ }^{131} \mathrm{Cs}$ ) and bi-radionuclide seed implants (Pd-I or Pd-Cs). A sensitivity analysis on the modeling parameters is also performed.

\section{Patients and Methods}

2.1. Treatment Plans. A source data file for the Prostate Seed Implant Dosimetry (PSID) Treatment Planning System (TPS) has been generated for InterSource seeds loaded with either ${ }^{103} \mathrm{Pd},{ }^{125} \mathrm{I},{ }^{131} \mathrm{Cs}$, Pd-I, or Pd-Cs following the AAPM TG43U1 formalism, which we have adapted for multiple radionuclide brachytherapy sources [23].

Three patients were planned using peripheral seed placement: patient 1 with the smallest prostate, patient 2 with a medium-sized prostate, and patient 3 with the largest prostate. Prostate and urethra volumes of each patient are given in Table 1. The urethral volume is defined as the volume enclosed by the urethra surface. Each patient has been planned with the five types of seeds. The prescription doses used for bi-radionuclide implants have been derived previously [24] and are summarized in Table 2 . The method that we used yields a single, fixed value of $142 \mathrm{~Gy}$ for the prescription dose of a Pd-I implant whereas it results in two different values for the Pd-Cs mixture: $115 \mathrm{~Gy}$ and $128 \mathrm{~Gy}$. However, these values are associated with large uncertainties. They were rounded to $145 \mathrm{~Gy}, 115 \mathrm{~Gy}$, and $130 \mathrm{~Gy}$, respectively, as safety margins on tumor control. The total number of treatment plans is therefore 18 (6 plans per patient).

2.2. Urethra NTCP. To the best of our knowledge, only Zaider et al. [1] have developed a model for the urethra NTCP after I-125 LDR. This model is based on a correlation between the probability of urethral toxicity and the dose received by the "hottest" $20 \%$ of the urethral volume $\left(\mathrm{DU}_{20}\right)$ after permanent prostate brachytherapy using ${ }^{125}$ I seeds. In order to apply such a model, and because in our study we are dealing with different radionuclides, we have converted the dose distribution for each implant to an equivalent I-125 LDR one. In order to do this, we derive the $\mathrm{DU}_{20-\mathrm{I}}$ of an I$125 \mathrm{LDR}$ treatment that would yield the same Biologically Effective Dose (BED) as the implant in question. The BED includes a linear and a quadratic term in dose. In the case of bi-radionuclide implant, its formulation requires the
TABLE 3: Modeling parameters and their absolute uncertainties. $p$ is the value of the considered parameter.

\begin{tabular}{lcccc}
\hline Parameter & Units & $p$ & $p-\Delta$ & $p+\Delta$ \\
\hline$\gamma$ & - & -2.60 & -3.10 & -2.10 \\
$\zeta$ & $\mathrm{Gy}$ & 0.0066 & 0.0050 & 0.0082 \\
$\mu$ & $\mathrm{h}^{-1}$ & 1.0 & 0 & 1.5 \\
$\alpha / \beta$ & $\mathrm{Gy}$ & 16.6 & 5 & 25 \\
$\mathrm{RBE}_{P d}$ & - & 1.41 & 1.00 & 1.75 \\
$\mathrm{RBE}_{I}$ & - & 1.28 & 1.00 & 1.45 \\
$\mathrm{RBE}_{C s}$ & - & 1.28 & 1.00 & 1.45 \\
$\delta_{1}$ & - & Variable & $\delta_{1}-0.020$ & $\delta_{1}+0.020$ \\
\hline
\end{tabular}

contribution of each radionuclide to the $\mathrm{DU}_{20}$. The models and procedure are described in what follows.

2.2.1. Logistic Model. Based on a logistic regression analysis of patients treated by I-125 LDR, Zaider et al. [1] inferred the probability of unresolved Grade 2 or higher urethral toxicity at 12 months as a function of $\mathrm{DU}_{20}$ :

$$
\mathrm{NTCP}_{\text {ureth }}=P_{\text {tox }, 12}\left(\mathrm{DU}_{20-\mathrm{I}}\right)=\frac{1}{1+\exp \left[-\left(\gamma+\zeta \mathrm{DU}_{20-\mathrm{I}}\right)\right]},
$$

where $\gamma$ and $\zeta$ are two fitting parameters given with their uncertainties in Table 3. Further in the text, iodine-125 referring to the I-125 LDR equivalent implant will be noted I-125 whereas iodine-125 referring to our implants will be noted ${ }^{125} \mathrm{I}$.

2.2.2. I-125 LDR Equivalent $\mathrm{DU}_{20}$. The parameters of the urethral NTCP model correspond to an I-125 LDR treatment. Hence, the $\mathrm{DU}_{20}$ for the different other radionuclides or mixtures has to be converted to an I-125 LDR equivalent $\mathrm{DU}_{20}$. This conversion will be referred to below as "I125 conversion". The Biologically Effective Dose (BED) is a powerful tool for this purpose as it allows a comparison of different treatment modalities.

The BED for an LDR implant whose seeds contain one radionuclide is $[2,25]$

$$
\mathrm{BED}_{\mathrm{LDR}}=\mathrm{RBE}_{\max } \cdot \mathrm{DU}_{20}+\frac{1}{\alpha / \beta} \frac{\lambda\left(\mathrm{DU}_{20}\right)^{2}}{(\lambda+\mu)},
$$

where $\mathrm{RBE}_{\max }$ is the Relative Biological Effectiveness of the radionuclide at very low-dose rate; $[2] ; \alpha / \beta$ is the ratio of radiosensitivity parameters; $\lambda$ is the radioactive decay constant of the radionuclide; $\mu$ is the sublethal cell damage repair rate. For the urethra, the commonly used values for $\alpha / \beta$ and $\mu$ are $3 \mathrm{~Gy}$ and $0.5 \mathrm{~h}^{-1}$ respectively. For normal tissues, cell repopulation is not taken into account. The $\mathrm{RBE}_{\max }$ values used in this study are from Wang et al. [26]: 1.41 for ${ }^{103} \mathrm{Pd}$ and 1.28 for ${ }^{125} \mathrm{I}$. Due to the lack of experimental data on the $\mathrm{RBE}_{\max }$ of ${ }^{131} \mathrm{Cs}$, its value has been set to the same value (i.e., 1.28) as that for ${ }^{125}$ I. This assumption is based on the fact that their emission spectra are similar. Note that (2) differs from the expression in 
Zaider et al. [1] one. However, as the sublethal cell damage repair rate for the urethra is much larger than the radioactive decay constant, both results fit within $1 \%$ in the worst case.

The clinical outcome would be equivalent for two treatments if they yield the same BED:

$$
\begin{gathered}
\mathrm{BED}_{\mathrm{LDR}-x}=\mathrm{BED}_{\mathrm{LDR}-\mathrm{I}}, \\
\mathrm{RBE}_{\max -x} \cdot \mathrm{DU}_{20-x}+\frac{1}{\alpha / \beta} \frac{\lambda_{x}\left(\mathrm{DU}_{20-x}\right)^{2}}{\left(\lambda_{x}+\mu\right)} \\
=\mathrm{RBE}_{\max -\mathrm{I}} \cdot \mathrm{DU}_{20-\mathrm{I}}+\frac{1}{\alpha / \beta} \frac{\lambda_{I}\left(\mathrm{DU}_{20-\mathrm{I}}\right)^{2}}{\left(\lambda_{\mathrm{I}}+\mu\right)} .
\end{gathered}
$$

The I-125 LDR equivalent $\mathrm{DU}_{20-\mathrm{I}}$ of the $\mathrm{DU}_{20-x}$ resulting from an implant based on radionuclide $x$ can be calculated by expressing (4) as a quadratic

$$
A\left[\mathrm{DU}_{20-\mathrm{I}}\right]^{2}+B \cdot\left[\mathrm{DU}_{20-\mathrm{I}}\right]+C=0,
$$

where

$$
\begin{gathered}
A=\frac{\lambda_{\mathrm{I}}}{\left(\lambda_{\mathrm{I}}+\mu\right)}, \\
B=\left(\frac{\alpha}{\beta}\right) \mathrm{RBE}_{\max -\mathrm{I}} \\
C=-\left[\left(\frac{\alpha}{\beta}\right) \mathrm{RBE}_{\max -x} \cdot \mathrm{DU}_{20-x}+\frac{\lambda_{x}\left(\mathrm{DU}_{20-x}\right)^{2}}{\left(\lambda_{x}+\mu\right)}\right] .
\end{gathered}
$$

As $\mathrm{DU}_{20-\mathrm{I}}, A$ and $B$ must have positive values, the only valid solution of (5) is

$$
\mathrm{DU}_{20-\mathrm{I}}=\frac{-B+\sqrt{B^{2}-4 A C}}{2 A} .
$$

For a bi-radionuclide seed implant, the solution is the same as (9) with same expression for $A$ and $B$, but the expression of $C$ is

$$
\begin{aligned}
C=-\left[\left(\frac{\alpha}{\beta}\right) \mathrm{DU}_{20} \sum_{i=1}^{2} \delta_{i} \cdot \mathrm{RBE}_{\max -i}\right. \\
\left.\quad+2\left(\mathrm{DU}_{20}\right)^{2} \sum_{i=1}^{2} \sum_{j=1}^{2} \frac{\lambda_{i} \lambda_{j} \delta_{i} \delta_{j}}{\left(\lambda_{i}+\mu\right)\left(\lambda_{i}+\lambda_{j}\right)}\right],
\end{aligned}
$$

where $\mathrm{DU}_{20}$ is the one of the bi-radionuclide implant and $\delta_{i}$ is the contribution of radionuclide $i$ in the seed to the $\mathrm{DU}_{20}$.

2.2.3. Bi-radionuclide Case: $\delta_{i}$ Contributions. Equation (10) requires that the contribution $\delta_{i}$ of each radionuclide to $\mathrm{DU}_{20}$ be known for each point in space that is considered. Treatment plans show that the relative contribution of each radionuclide can be considered as constant throughout the urethral volume. Hence, everywhere in the urethra:

$$
D=D_{1}+D_{2}=D\left(\delta_{1}+\delta_{2}\right)=D\left(\delta_{1}+\left(1-\delta_{1}\right)\right) .
$$

Let us include the RBE effect directly in the TPS file that defines the seed data (dose distribution in the adapted AAPM TG43U1 dosimetry formalism [23]). This can be done by modifying the adapted radial dose function and 1Danisotropy function of the seed (see Appendix). The dose distribution $D_{\mathrm{RBE}}$ in the organs provided by the TPS would therefore also include the RBE effect:

$$
\begin{aligned}
D_{\mathrm{RBE}} & =D_{1} \mathrm{RBE}_{\max -1}+D_{2} \mathrm{RBE}_{\max -2}, \\
& =D\left(\delta_{1} \mathrm{RBE}_{\max -1}+\delta_{2} \mathrm{RBE}_{\max -2}\right), \\
D_{\mathrm{RBE}} & =D\left(\delta_{1} \mathrm{RBE}_{\max -1}+\left(1-\delta_{1}\right) \mathrm{RBE}_{\max -2}\right) .
\end{aligned}
$$

As the $\delta_{i}$ 's are spatially independent, (13) can be applied directly to the $\mathrm{DU}_{20}$ value. Therefore, if the $\mathrm{DU}_{20}$ from the treatment plan with and without RBE effect $\left(D^{20-R B E}\right.$ and $\mathrm{DU}_{20}$, resp.,) are known, then the contribution of each radionuclide to $\mathrm{DU}_{20}$ can be computed from

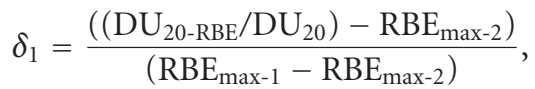

$$
\begin{aligned}
& \delta_{2}=1-\delta_{1} .
\end{aligned}
$$

These values turn out to match within $1 \%$ the mean $\delta_{i}$ 's that one would obtain at the middle of the urethra contour on each TRUS image slice. This result is not surprising as the urethra is approximately in the middle of the prostate and therefore in the middle of the seed distribution. All the seeds of the implant contribute with different magnitudes to urethral dose, providing a homogeneous dose distribution throughout the urethra for each radionuclide.

2.2.4. Propagation of Uncertainty. The method described in the above three sections includes many parameters associated with uncertainties. The uncertainties in $\mathrm{DU}_{20-\mathrm{I}}$ come from the radiobiological parameters $(\mu, \alpha / \beta)$ and the $\mathrm{RBE}_{\max }$ of each radionuclide. These uncertainties along with those of the NTCP fitting parameters will affect the urethral NTCP. The NTCP fitting parameters' uncertainties will not affect the relative NTCPs of the different seed implants as the fitting parameters are not radionuclide dependent. By contrast, $\mathrm{DU}_{20 \text {-I }}$ parameters are radionuclide dependent. Therefore, the repercussion of the $\mathrm{DU}_{20-\mathrm{I}}$ parameters' uncertainties on $\mathrm{DU}_{20-\mathrm{I}}$ and the subsequent repercussion on urethral NTCP were studied.

The $\mathrm{DU}_{20-\mathrm{I}}$ uncertainty can be expressed as follows:

$$
\begin{aligned}
\Delta \mathrm{DU}_{20-\mathrm{I}}= & \left|\frac{\partial \mathrm{DU}_{20-\mathrm{I}}}{\partial \mu}\right| \Delta \mu+\left|\frac{\partial \mathrm{DU}_{20-\mathrm{I}}}{\partial(\alpha / \beta)}\right| \Delta(\alpha / \beta) \\
& +\left|\frac{\partial \mathrm{DU}_{20-\mathrm{I}}}{\partial \delta_{1}}\right| \Delta \delta_{1} \\
& +\mid \frac{\partial \mathrm{DU}_{20-\mathrm{I}}}{\partial \mathrm{RBE}_{\mathrm{I}-125}} \Delta \mathrm{RBE}_{\mathrm{I}-125}+\frac{\partial \mathrm{DU}_{20-\mathrm{I}}}{\partial \mathrm{RBE}_{\mathrm{I}}} \triangle \mathrm{RBE}_{1} \\
& +\frac{\partial \mathrm{DU}_{20-\mathrm{I}}}{\partial \mathrm{RBE}_{2}} \Delta \mathrm{RBE}_{2} \mid .
\end{aligned}
$$


The uncertainty on RBE values contains three different terms: the uncertainty on I-125 RBE and on the RBE of each radionuclide in the seed. Note that in the case of monoradionuclide implants, $\partial \mathrm{DU}_{20-\mathrm{I}} / \partial \mathrm{RBE}_{2}=0$ and $\Delta \delta=0$. If the implant uses ${ }^{125} \mathrm{I}$ or ${ }^{131} \mathrm{Cs}$, their $\mathrm{RBE}_{\max }$ value has to be the same as the I-125 $\mathrm{RBE}_{\max }$ value for consistency reasons. RBE uncertainties are therefore correlated and the absolute value has to be taken over the whole RBE uncertainties. Moreover, the palladium-103 RBE is correlated to the iodine-125 RBE. Depending on the study $[26,27]$, if a high value of iodine$125 \mathrm{RBE}_{\max }$ (1.45 instead of 1.28) is chosen then a high value of palladium-103 $\mathrm{RBE}_{\max }$ is obtained (1.75 instead of 1.41). As a result, the uncertainties on ${ }^{103} \mathrm{Pd} \mathrm{RBE}$ are correlated to the uncertainties on iodine-125 RBE, which can be rewritten as

$$
\triangle \mathrm{RBE}_{\mathrm{Pd}}=\triangle \mathrm{RBE}_{\mathrm{I}}\left(\frac{d \mathrm{RBE}_{\mathrm{Pd}}}{d \mathrm{RBE}_{\mathrm{I}}}\right) .
$$

The term into bracket has been calculated using the above mentioned value of ${ }^{103} \mathrm{Pd}$ and ${ }^{125} \mathrm{IRBE}_{\max }$ values and is equal to 1.65 . terms:

The NTCP uncertainty expression includes only three

$$
\begin{aligned}
\Delta \mathrm{NTCP}= & \left|\frac{\partial \mathrm{NTCP}}{\partial \gamma}\right| \Delta \gamma+\left|\frac{\partial \mathrm{NTCP}}{\partial \zeta}\right| \Delta \zeta \\
& +\left|\frac{\partial \mathrm{NTCP}}{\partial \mathrm{DU}_{20-\mathrm{I}}}\right| \triangle \mathrm{DU}_{20-\mathrm{I} \cdot}
\end{aligned}
$$

The partial derivatives of $\mathrm{DU}_{20-\mathrm{I}}$ and NTCP are given in Appendices B and C, respectively.

\section{Results}

3.1. Modeling Parameters and Associated Uncertainties. The radiobiological parameters used in this study are limited to the $\alpha / \beta$ ratio and sublethal cell damage repair rate, $\mu$. First, we choose the values that Zaider et al. used in their study. These are the one reported in Table 3 . This high $\alpha / \beta$ ratio is typical for tumors (typical range 5-25 Gy) but does not correspond to the commonly used value for late responding normal tissue (typical range 1-5 Gy) [2]. Therefore, we also calculate the DU 20 -I and NTCP with $\alpha / \beta=3 \mathrm{~Gy}(1-5 \mathrm{~Gy})$ and $\mu=0.5 \mathrm{~h}^{-1}\left(0-1.5 \mathrm{~h}^{-1}\right)$ reported in the Dale and Jones text book [2]. The values into brackets are the uncertainty range used for the uncertainty calculations.

Relative Biological Effectiveness values are the one published by Wang et al. [26]. Due to the lack of experimental data on the $\mathrm{RBE}_{\max }$ of ${ }^{131} \mathrm{Cs}$, its value has been set to the same value as the one of ${ }^{125} \mathrm{I}$. The uncertainty interval is based on RBE values published by other authors. The minimum $\mathrm{RBE}_{\text {max }}$ value is 1 by definition. The maximum $\mathrm{RBE}_{\text {max }}$ values have been set to the ones published by Antipas et al. [27].

Finally, the uncertainty on $\delta_{1}$, the contribution of the first radionuclide to the $\mathrm{DU}_{20}$, is equal to the maximal deviation with respect to the mean value of the $\delta_{1}$ obtained in the middle of the urethra on TRUS slices.

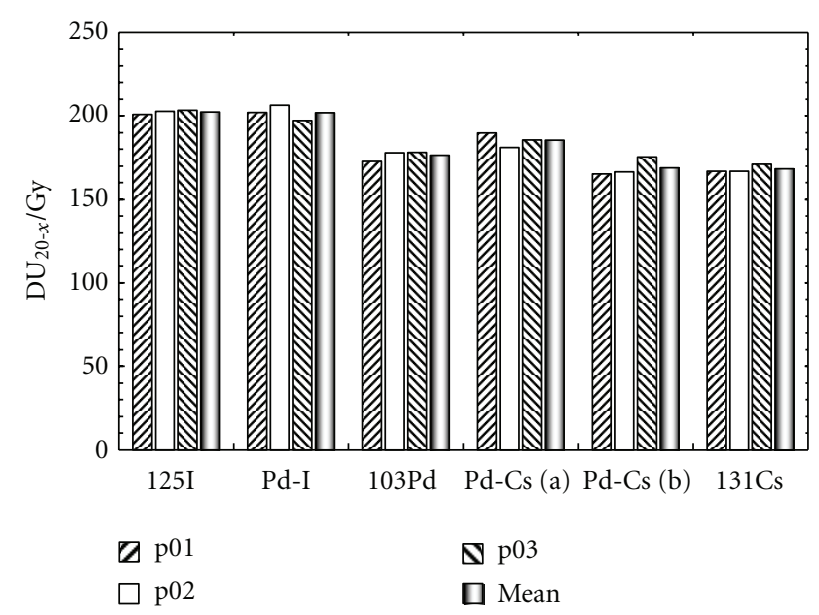

FIGURE 1: $\mathrm{DU}_{20}$ for the different seeds (mono- and bi-radionuclide) and patients. The $\mathrm{DU}_{20}$ averaged over the three patients is also plotted.

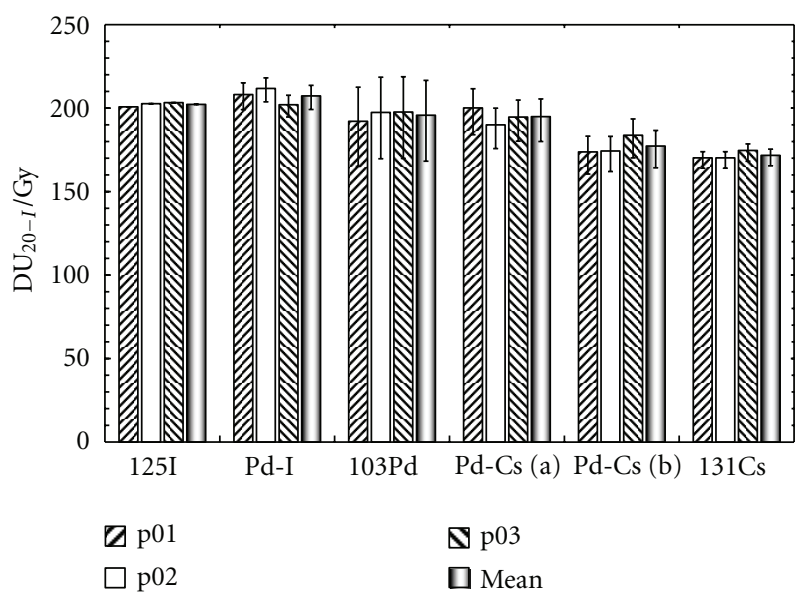

FIGURE 2: I-125 LDR equivalent $D_{20-I}$ results for the different implants (mono- and bi-radionuclide) and patients. The mean $\mathrm{DU}_{20-\mathrm{I}}$ over the three patients is also plotted. Zaider et al. radiobiological parameters are used. Error bars were calculated using (15).

3.2. $\mathrm{DU}_{20}$ and I-125 LDR Equivalent $\mathrm{DU}_{20}, \mathrm{DU}_{20-\mathrm{I}}$. The TPS $\mathrm{DU}_{20}$ output for the different patients and radionuclides are presented in Figure 1.

The mean $\mathrm{DU}_{20}$ value is also shown. As discussed above, these values are not comparable as they do not correspond to the same delivery scheme. Nevertheless, it will be interesting to observe how much the $\mathrm{DU}_{20}$ is modified for each seed implant after the I-125 conversion. It is emphasized that the $\mathrm{DU}_{20}$ does not change significantly from one patient to another one.

The implants can be compared after the I-125 conversion. The $\mathrm{DU}_{20}$ of each seed implant has been converted to an I-125 LDR equivalent $\mathrm{DU}_{20}$ using (6) to (10) and (14). Figure 2 shows the results and the numerical values are reported in Table 4.

The effect of this conversion is to increase the $\mathrm{DU}_{20}$. The smallest increase is observed for the ${ }^{131} \mathrm{Cs}$ implant with 3.3 Gy and the largest one for the ${ }^{103} \mathrm{Pd}$ seed implant with 
TABle 4: Dose received by the hottest $20 \%$ of the urethra volume $\left(\mathrm{DU}_{20}\right), 125 \mathrm{I} \mathrm{LDR}$ equivalent $\mathrm{DU}_{20}\left(\mathrm{DU}_{20-\mathrm{I}}\right)$, and urethra Normal Tissue Complication Probability (NTCP) for the different seed implants. The values are averaged over the three patients. Radiobiological parameters from Zaider et al. are used.

\begin{tabular}{lccc}
\hline & $\mathrm{DU}_{20}(\mathrm{~Gy})$ & $\mathrm{DU}_{20-\mathrm{I}}(\mathrm{Gy})$ & $\mathrm{NTCP}(\%)$ \\
\hline${ }^{125} \mathrm{I}$ & 202.3 & 202.3 & 22.0 \\
$\mathrm{Pd}-\mathrm{I}$ & 201.8 & 207.4 & 22.6 \\
${ }^{103} \mathrm{Pd}$ & 176.3 & 195.8 & 21.3 \\
Pd-Cs (a) & 185.5 & 194.9 & 21.2 \\
Pd-Cs (b) & 169.0 & 177.3 & 19.3 \\
${ }^{131} \mathrm{Cs}$ & 168.4 & 171.7 & 18.7 \\
\hline
\end{tabular}

19.5 Gy. The uncertainty associated with these values will be the subject of a separate point. As expected, ${ }^{125} \mathrm{I}$ is not affected by the I-125 conversion. The ${ }^{131} \mathrm{Cs}$ implant is also not significantly affected by the I- 125 conversion which suggests that the quadratic contribution of BED is small.

3.3. Urethral NTCP. Although the $\mathrm{DU}_{20-\mathrm{I}}$ figures correspond to the same delivery scheme (I-125 LDR) and they can be used to compare treatments, they do not give information about the probability of urethral complications. These probabilities have been computed using (1) and are plotted in Figure 3. Numerical values are reported in Table 4.

The NTCPs are larger than the one published by Zaider et al. who obtained NTCPs of about $16 \%$ for 143 Gy I-125 LDR [1]. However, a more recent study by Zelefsky et al. reports $19 \%$ of patient experiencing late Grade 2 urinary symptoms [28].

All the NTCP results fall in the 19 to $23 \%$ range. The comparison between these data is therefore very difficult as these results are affected by large uncertainties. Their origin is discussed in the next section.

3.4. Uncertainties. We have calculated the contribution of each parameters uncertainty to the total $\mathrm{DU}_{20-\mathrm{I}}$ and NTCP uncertainties. The values are tabulated in Tables 5 and 6, respectively.

Firstly, the results show that the $\mathrm{DU}_{20-\mathrm{I}}$ uncertainty is mainly due to the uncertainty on the $\mathrm{RBE}_{\max }$ value. The uncertainty is the greatest for ${ }^{103} \mathrm{Pd}$ and the least for ${ }^{131} \mathrm{Cs}$ apart from ${ }^{125} \mathrm{I}$ which is not affected by the conversion and is not subjected to model-related errors. Sublethal damage repair rate, $\mu$, as well as ratio $\alpha / \beta$ have little effect on the $\mathrm{DU}_{20-\mathrm{I}}$ value.

Secondly, the urethral NTCP uncertainties are mainly related to the parameter-fitting uncertainties. The error produced by $\triangle \mathrm{DU}_{20-\mathrm{I}}$ has a secondary importance. The total relative uncertainty is therefore almost constant among the different modalities, ranging from 12 to $17 \%$.

However, these figures also depend on the radiobiological data chosen for the calculations. If the Dale and Jones radiobiological parameters $(\alpha / \beta$ and $\mu)$ are used, the uncertainty generated by the sublethal damage repair rate becomes the most important one. The $\mathrm{DU}_{20-\mathrm{I}}$ and urethral

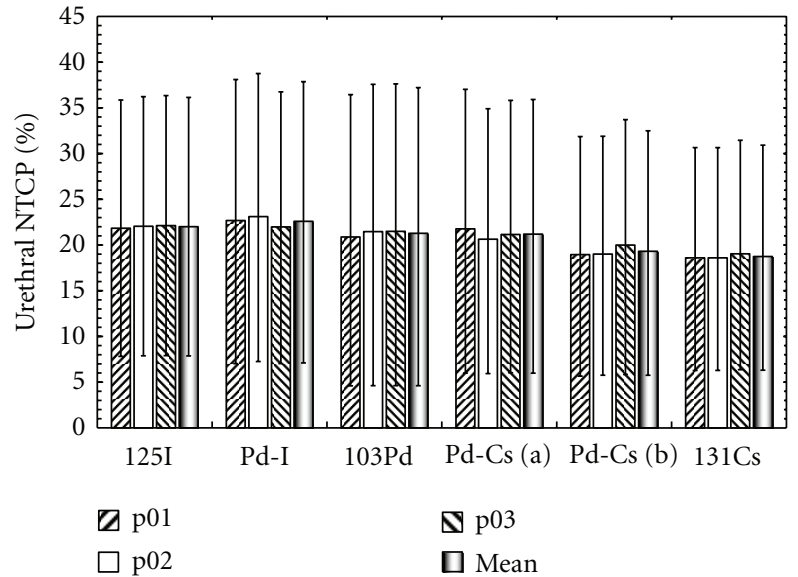

Figure 3: Urethral NTCP results for each patient and each implant (mono- and bi-radionuclide). The mean NTCP over the three patients is also plotted for each implant. Zaider et al. radiobiological parameters are used. Error bars were calculated using (17).

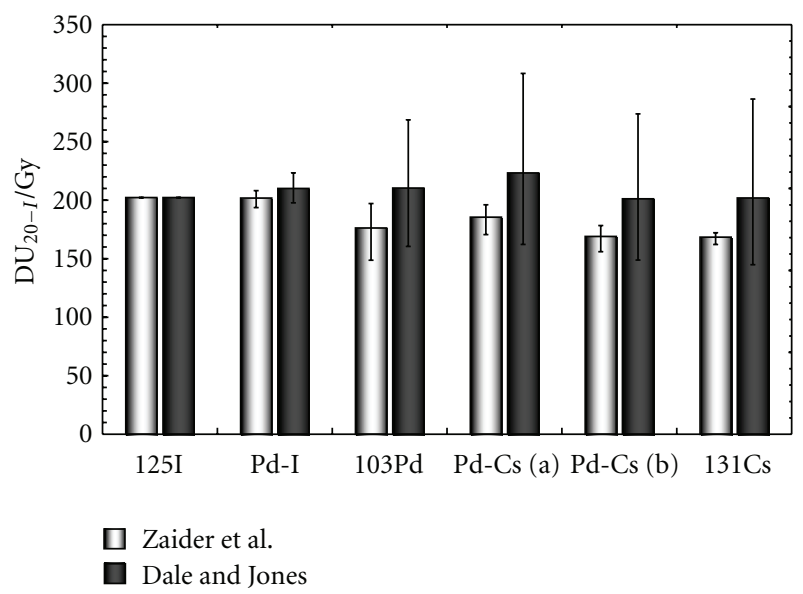

Figure 4: Comparison of $\mathrm{DU}_{20-\mathrm{I}}$ for two different values of $\alpha / \beta$ and $\mu: \alpha / \beta=16.6$ Gy and $\mu=1 \mathrm{~h}^{-1}$ (Zaider et al. [1]) and $\alpha / \beta=3 \mathrm{~Gy}$ and $\mu=0.5 \mathrm{~h}^{-1}$ (Dale and Jones [2]). Error bars were calculated using (15).

NTCP obtained with Zaider et al. and Dale and Jones radiobiological parameters are compared in Figures 4 and 5, respectively.

${ }^{131} \mathrm{Cs}$ is the most affected radionuclide. RBE uncertainty remains almost unchanged. Uncertainties produced by $\delta_{i}$ contributions are still negligible compared to other parameter's uncertainties. These errors will have repercussions on NTCP uncertainties with the largest effect for ${ }^{131}$ Cs. The contribution of the total $\triangle \mathrm{DU}_{20-\mathrm{I}}$ on the NTCP total uncertainty is now of the same order of magnitude as the one produced by the parameter-fitting error.

\section{Discussion and Conclusions}

The variation in the $\mathrm{DU}_{20}$ due to $\mathrm{I}-125$ conversion is influenced by two major contributions. First, the linear term in the BED expression depends only on the $\mathrm{RBE}_{\max }$ value. Second, the quadratic term is more complex and results from 
TABLE 5: $\mathrm{DU}_{20-\mathrm{I}}$ uncertainty produced by each parameter and total $\mathrm{DU}_{20-\mathrm{I}}$ uncertainty for each implant using Zaider et al. radiobiological parameters.

\begin{tabular}{|c|c|c|c|c|c|c|c|}
\hline \multicolumn{2}{|c|}{$\triangle \mathrm{DU}_{20-\mathrm{I}}(\mathrm{Gy})$} & ${ }^{125} \mathrm{I}$ & Pd-I & ${ }^{103} \mathrm{Pd}$ & Pd-Cs (a) & Pd-Cs (b) & ${ }^{131} \mathrm{Cs}$ \\
\hline \multirow{2}{*}{$\mu$} & $+\Delta$ & 0.0 & 0.1 & 0.8 & 1.6 & 1.3 & 1.6 \\
\hline & $-\Delta$ & 0.0 & 0.3 & 1.6 & 3.1 & 2.6 & 3.3 \\
\hline \multirow{2}{*}{$\alpha / \beta$} & $+\Delta$ & 0.0 & 0.1 & 0.8 & 1.6 & 1.3 & 1.7 \\
\hline & $-\Delta$ & 0.0 & 0.2 & 1.1 & 2.2 & 1.8 & 2.3 \\
\hline \multirow{2}{*}{$\delta_{1}$} & $+\Delta$ & 0.0 & 0.4 & 0.0 & 0.3 & 0.3 & 0.0 \\
\hline & $-\Delta$ & 0.0 & 0.4 & 0.0 & 0.3 & 0.3 & 0.0 \\
\hline \multirow{2}{*}{$\mathrm{RBE}$ (tot) } & $+\Delta$ & 0.0 & 5.6 & 19.3 & 7.1 & 6.4 & 0.4 \\
\hline & $-\Delta$ & 0.0 & 7.2 & 24.8 & 9.3 & 8.3 & 0.7 \\
\hline \multirow{2}{*}{ Total } & $+\Delta$ & 0.0 & 6.3 & 20.9 & 10.6 & 9.3 & 3.7 \\
\hline & $-\Delta$ & 0.0 & 8.1 & 27.5 & 14.9 & 13.0 & 6.3 \\
\hline
\end{tabular}

TABLE 6: NTCP uncertainty produced by each parameter and total NTCP uncertainty for each implant using Zaider et al. radiobiological parameters.

\begin{tabular}{|c|c|c|c|c|c|c|c|}
\hline \multicolumn{2}{|c|}{$\triangle$ NTCP $(\%)$} & ${ }^{125} \mathrm{I}$ & Pd-I & ${ }^{103} \mathrm{Pd}$ & Pd-Cs (a) & Pd-Cs (b) & ${ }^{131} \mathrm{Cs}$ \\
\hline$\gamma$ & $\pm \Delta$ & $8.6 \%$ & $8.7 \%$ & $8.4 \%$ & $8.4 \%$ & $7.8 \%$ & $7.6 \%$ \\
\hline$\zeta$ & $\pm \triangle$ & $5.6 \%$ & $5.8 \%$ & $5.2 \%$ & $5.2 \%$ & $4.4 \%$ & $4.2 \%$ \\
\hline \multirow{2}{*}{$\mathrm{DU}_{20-\mathrm{I}}$} & $+\triangle$ & $0.0 \%$ & $0.7 \%$ & $2.3 \%$ & $1.2 \%$ & $1.0 \%$ & $0.4 \%$ \\
\hline & $-\Delta$ & $0.0 \%$ & $0.9 \%$ & $3.0 \%$ & $1.6 \%$ & $1.3 \%$ & $0.6 \%$ \\
\hline \multirow{2}{*}{ Total } & $+\Delta$ & $14.1 \%$ & $15.3 \%$ & $15.9 \%$ & $14.7 \%$ & $13.2 \%$ & $12.2 \%$ \\
\hline & $-\Delta$ & $14.1 \%$ & $15.5 \%$ & $16.7 \%$ & $15.2 \%$ & $13.6 \%$ & $12.4 \%$ \\
\hline
\end{tabular}

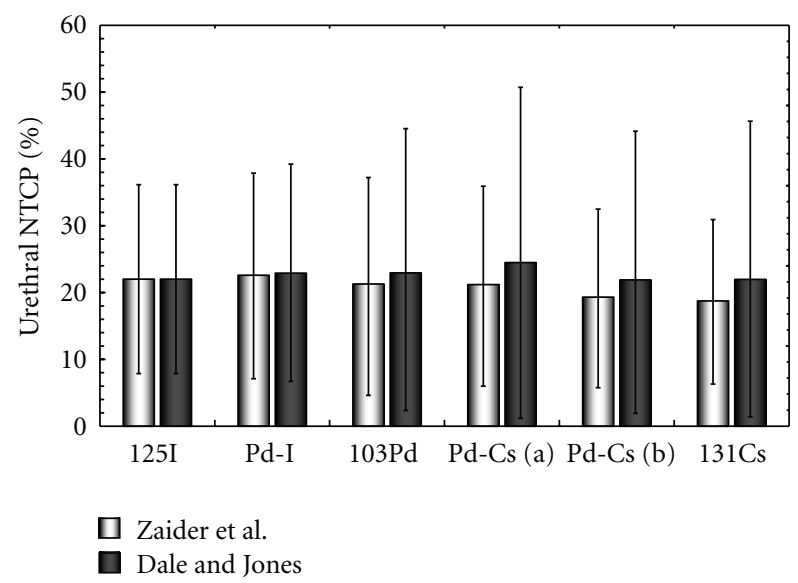

Figure 5: Comparison of urethral NTCP for two different values of $\alpha / \beta$ and $\mu: \alpha / \beta=16.6$ Gy and $\mu=1 h^{-1}$ (Zaider et al. [1]) and $\alpha / \beta=3$ Gy and $\mu=0.5 \mathrm{~h}^{-1}$ (Dale and Jones [2]). Error bars were calculated using (17).

the influence of three separate parameters: $\alpha / \beta$ and $\mu$ which are tissue dependent and the radionuclide half-life.

Firstly, the ${ }^{125} \mathrm{I} \mathrm{DU}_{20}$ will not be affected by the I-125 conversion as only one radionuclide is involved. Secondly, as one chooses the same $\mathrm{RBE}_{\max }$ value for ${ }^{131} \mathrm{Cs}$ and ${ }^{125} \mathrm{I}$, the linear term of the ${ }^{131} \mathrm{Cs}$ BED is not affected by the ${ }^{125} \mathrm{I}$ conversion. Since the value of $\alpha / \beta$ chosen by Zaider et al. is large, the quadratic contribution to the BED is small and influences the ${ }^{131} \mathrm{Cs} \mathrm{BED}$ by only $2 \%$. If future experiments provide a $\mathrm{RBE}_{\max }$ value for ${ }^{131} \mathrm{Cs}$ that is different from that of ${ }^{125} \mathrm{I}$, larger variation in $\mathrm{I}-125$ equivalent $\mathrm{DU}_{20}$ may be observed. Thirdly, ${ }^{103} \mathrm{Pd}$ has a larger $\mathrm{RBE}_{\max }$ value than ${ }^{125} \mathrm{I}$. Its linear contribution to BED will therefore also contribute to a modified value of the $\mathrm{DU}_{20}$. This contribution of the quadratic term accounts for only $1 \%$ of the total BED. The difference in $\mathrm{RBE}_{\max }$ value between ${ }^{103} \mathrm{Pd}$ and I-125 is therefore the main cause of the large increase in $\mathrm{DU}_{20}$ after I-125 conversion. Finally, the changes in the Pd-I and Pd-Cs $\mathrm{DU}_{20}$ due to I-125 conversion are related to both the linear and the quadratic contribution of each radionuclide to BED. Pd-Cs is the most affected mixture as both radionuclides differ from the one used for modeling (I-125).

The very similar values obtained for the NTCP and their large uncertainties makes it difficult, if not impossible, to conclude definitively whether, for equal tumour effect, bi-radionuclide brachytherapy would reduce the urethral complication probability relative to mono-radionuclide brachytherapy. Planning more patients would not improve the situation as these uncertainties are mainly due to the fitting parameter uncertainties of the empirical model of Zaider et al.

The large value assumed by Zaider et al. for the $\alpha / \beta$ ratio reduces the influence of the quadratic term on the total BED considerably. Therefore the sublethal damage repair $\mu$ on the BED will show its effect only for radionuclides with short half-lives (like ${ }^{131} \mathrm{Cs}$ ). This is also proven by the low 
$\mathrm{DU}_{20-\mathrm{I}}$ uncertainty associated with $\mu$ and to the $\alpha / \beta$ ratio. The uncertainty related to the $\delta_{1}$ also indicates that our approximation will not significantly affect the final results.

However, the Zaider et al. radiobiological parameters are not the only ones in common use. The results obtained with the radiobiological parameters from Dale and Jones (Figures 4 and 5) clearly show that such parameters could also have a large impact on the final NTCP value. Moreover, the lower value of $\alpha / \beta$ ratio increases the quadratic contribution of the BED, leading to larger uncertainties associated with $\alpha / \beta$ and $\mu$.

Finally, complication probabilities are certainly dependent on the way the treatment planning is performed. A logistic regression among patients treated by the each institution could also provide different NTCP parameter values. However, this would not affect the $\mathrm{DU}_{20-\mathrm{I}}$ results.

We can conclude that the use of the urethra NTCP model for biologically based treatment planning in permanent seed prostate brachytherapy requires either better fitting parameters (with less associated errors) or a different NTCP model (different morbidity indicator than $\mathrm{DU}_{20}$ e.g.).

\section{Appendix}

\section{A. Inclusion of RBE in TPS Dose Distribution}

In order to include the RBE effect in the TPS dose distribution, the radial dose function and the $1 \mathrm{D}$ or $2 \mathrm{D}$ anisotropy functions have to be modified. This modification is trivial in the case of mono-radionuclide brachytherapy: the radial dose function is multiplied by the radionuclide $\mathrm{RBE}_{\max }$ value and the anisotropy functions are not altered. In the case of bi-radionuclide implants, the $\mathrm{RBE}_{\max }$ value will be different from one radionuclide to another. Therefore,

$$
\begin{aligned}
g_{\mathrm{RBE}}(r) & =\sum_{i=1}^{2} c_{i} \mathrm{RBE}_{\max -i} g_{i}(r), \\
\phi_{\mathrm{an}-\mathrm{RBE}}(r) & =\frac{\sum_{i=1}^{2} c_{i} \mathrm{RBE}_{\max -i} g_{i}(r) \phi_{\mathrm{an}-i}(r)}{\sum_{i=1}^{2} c_{i} \mathrm{RBE}_{\max -i} g_{i}(r)}, \\
F(r, \theta) & =\frac{\sum_{i=1}^{2} c_{i} \mathrm{RBE}_{\max -i} g_{i}(r) F_{i}(r, \theta)}{\sum_{i=i}^{2} c_{i} \mathrm{RBE}_{\max -i} g_{i}(r)} .
\end{aligned}
$$

It can be shown that if (A.1) replaces (11), (14) and (16) in (4) or (5) of Nuttens and Lucas [23], this would give

$$
D(r, \theta)=\sum_{i=1}^{2} \mathrm{RBE}_{\max -i} D_{i}(r, \theta),
$$

which means that the TPS dose distribution includes well the RBE effect.

\section{B. Partial Derivatives of $\mathbf{D U _ { 2 0 - I }}$}

$\mathrm{DU}_{20-\mathrm{I}}$ is expressed as a function of $A, B$, and $C$ ((6) to (10)). Its derivative with respect to parameter $p$ can also be expressed as a function of $A, B$, and $C$ and their derivative with respect to parameter $p$ noted $(d A / d p)=\dot{A},(d B / d p)=$ $\dot{B}$ and $(d C / d p)=\dot{C}$

$$
\begin{aligned}
\frac{d \mathrm{DU}_{20-\mathrm{I}}}{d p}= & \frac{1}{2 A}\left(-\dot{B}+\frac{B \dot{B}-2 \dot{A} C-2 A \dot{C}}{\sqrt{B^{2}-4 A C}}\right) \\
& -\frac{-B+\sqrt{B^{2}-4 A C}}{2 A^{2}} \dot{A} .
\end{aligned}
$$

The expressions of $\dot{A}, \dot{B}$, and $\dot{C}$ are now given for each parameter $p$.

(1) $\mathbf{p}=\boldsymbol{\mu}$,

$$
\begin{aligned}
& \dot{A}=\frac{d A}{d \mu}=\frac{-\lambda_{\mathrm{I}}}{\left(\lambda_{\mathrm{I}}+\mu\right)^{2}}=\frac{-A^{2}}{\lambda_{\mathrm{I}}}, \\
& \dot{B}=\frac{d B}{d \mu}=0, \\
& \dot{C}=\frac{d C}{d \mu}=2 \sum_{i, j=1}^{2} \frac{\lambda_{i} \lambda_{j} \delta_{i} \delta_{j} \mathrm{DU}_{20-x}^{2}}{\left(\lambda_{i}+\mu\right)^{2}\left(\lambda_{i}+\lambda_{j}\right)},
\end{aligned}
$$

(2) $\mathbf{p}=(\boldsymbol{\alpha} / \boldsymbol{\beta})$,

$$
\begin{aligned}
& \dot{A}=\frac{d A}{d(\alpha / \beta)}=0, \\
& \dot{B}=\frac{d B}{d(\alpha / \beta)}=\mathrm{RBE}_{\mathrm{I}-125}, \\
& \dot{C}=\frac{d C}{d(\alpha / \beta)}=-\mathrm{DU}_{20-x} \sum_{i=1}^{2} \mathrm{RBE}_{i} \delta_{i},
\end{aligned}
$$

(3) $\mathbf{p}=\mathrm{RBE}_{\mathrm{I}-125}$,

$$
\begin{aligned}
& \dot{A}=\frac{d A}{d \mathrm{RBE}_{\mathrm{I}-125}}=0, \\
& \dot{B}=\frac{d B}{d \mathrm{RBE}_{\mathrm{I}-125}}=\frac{\alpha}{\beta}, \\
& \dot{C}=\frac{d C}{d \mathrm{RBE}_{\mathrm{I}-125}}=0,
\end{aligned}
$$

(4) $\mathbf{p}=\mathrm{RBE}_{\mathbf{i}}$,

$$
\begin{aligned}
& \dot{A}=\frac{d A}{d \mathrm{RBE}_{\mathrm{i}}}=0, \\
& \dot{B}=\frac{d B}{d \mathrm{RBE}_{\mathrm{i}}}=0 \\
& \dot{C}=\frac{d C}{d \mathrm{RBE}_{\mathrm{i}}}=-\left(\frac{\alpha}{\beta}\right) \mathrm{DU}_{20-x} \delta_{i},
\end{aligned}
$$

(5) $\mathbf{p}=\boldsymbol{\delta}_{1}$, 
These expressions apply only for the bi-radionuclide implant for which $\delta_{1} \neq 1$

$$
\begin{aligned}
\dot{A}= & \frac{d A}{d \delta_{1}}=0, \\
\dot{B}= & \frac{d B}{d \delta_{1}}=0, \\
\dot{C}= & \frac{d C}{d \delta_{1}} \\
= & -\left(\frac{\alpha}{\beta}\right) \mathrm{DU}_{20-x}\left(\mathrm{RBE}_{1}-\mathrm{RBE}_{2}\right) \\
& -2 \mathrm{DU} \mathrm{D}_{20-x}^{2}\left[\frac{\lambda_{1} \delta_{1}}{\left(\lambda_{1}+\mu\right)}+\frac{\lambda_{1} \lambda_{2}\left(1-2 \delta_{1}\right)}{\left(\lambda_{1}+\mu\right)\left(\lambda_{1}+\lambda_{2}\right)}\right. \\
& \left.\quad+\frac{\lambda_{1} \lambda_{2}\left(1-2 \delta_{1}\right)}{\left(\lambda_{2}+\mu\right)\left(\lambda_{1}+\lambda_{2}\right)}+\frac{\lambda_{2}\left(\delta_{1}-1\right)}{\left(\lambda_{2}+\mu\right)}\right] .
\end{aligned}
$$

\section{Partial Derivatives of NTCP}

The NTCP expression is given by (1) and its partial derivatives with respect to each parameter are

$$
\begin{aligned}
& \frac{\partial \mathrm{NTCP}}{\partial \gamma}=\frac{\exp \left[-\left(\gamma+\zeta \mathrm{DU}_{20-\mathrm{I}}\right)\right]}{\left\{1+\exp \left[-\left(\gamma+\zeta \mathrm{DU}_{20-\mathrm{I}}\right)\right]\right\}^{2}}, \\
& \frac{\partial \mathrm{NTCP}}{\partial \zeta}=\frac{\mathrm{DU}_{20-\mathrm{I}} \exp \left[-\left(\gamma+\zeta \mathrm{DU}_{20-\mathrm{I}}\right)\right]}{\left\{1+\exp \left[-\left(\gamma+\zeta \mathrm{DU}_{20-\mathrm{I}}\right)\right]\right\}^{2}}, \\
& \frac{\partial \mathrm{NTCP}}{\partial \mathrm{DU}_{20-\mathrm{I}}}=\frac{\zeta \exp \left[-\left(\gamma+\zeta \mathrm{DU}_{20-\mathrm{I}}\right)\right]}{\left\{1+\exp \left[-\left(\gamma+\zeta \mathrm{DU}_{20-\mathrm{I}}\right)\right]\right\}^{2}} .
\end{aligned}
$$

\section{Acknowledgments}

V. E. Nuttens was supported by a Grant from the Belgian "Fonds pour la formation à la Recherche dans l'Industrie et dans l'Agriculture" (FRIA). Special thanks are due to H. C. Winter, C. D. Lee (Clatterbridge Center for Oncology) for helpful discussions.

\section{References}

[1] M. Zaider, M. J. Zelefsky, G. N. Cohen et al., "Methodology for biologically-based treatment planning for combined lowdose-rate (permanent implant) and high-dose-rate (fractionated) treatment of prostate cancer," International Journal of Radiation Oncology Biology Physics, vol. 61, no. 3, pp. 702-713, 2005.

[2] R. G. Dale and B. Jones, Radiobiological Modelling in Radiation Oncology, British Institute of Radiology, London, UK, 2007.

[3] A. Niemierko and M. Goitein, "Implementation of a model for estimating tumor control probability for an inhomogeneously irradiated tumor," Radiotherapy and Oncology, vol. 29, no. 2, pp. 140-147, 1993.

[4] S. Webb and A. E. Nahum, "A model for calculating tumour control probability in radiotherapy including the effects of inhomogeneous distributions of dose and clonogenic cell density," Physics in Medicine and Biology, vol. 38, no. 6, pp. 653666, 1993.

[5] B. Sanchez-Nieto and A. E. Nahum, "Bioplan: software for the biological evaluation of radiotherapy treatment plans," Medical Dosimetry, vol. 25, no. 2, pp. 71-76, 2000.

[6] A. Niemierko, M. Urie, and M. Goitein, "Optimization of 3D radiation therapy with both physical and biological end points and constraints," International Journal of Radiation Oncology Biology Physics, vol. 23, no. 1, pp. 99-108, 1992.

[7] Y. Kim and W. A. Tomé, "Risk-adaptive optimization: selective boosting of high-risk tumor subvolumes," International Journal of Radiation Oncology Biology Physics, vol. 66, no. 5, pp. 1528-1542, 2006.

[8] Z. Malik, C. Eswar, J. Dobson, J. Fenwick, and A. Nahum, "IsoNTCP customization of the prescription dose in lung-tumour radiotherapy," Radiotherapy and Oncology, vol. 84, pp. S278S279, 2007.

[9] A. Haworth, M. Ebert, D. Waterhouse, D. Joseph, and G. Duchesne, "Assessment of i-125 prostate implants by tumor bioeffect," International Journal of Radiation Oncology Biology Physics, vol. 59, no. 5, pp. 1405-1413, 2004.

[10] A. Haworth, M. Ebert, D. Waterhouse, D. Joseph, and G. Duchesne, "Prostate implant evaluation using tumour control probability-the effect of input parameters," Physics in Medicine and Biology, vol. 49, no. 16, pp. 3649-3664, 2004.

[11] W. D. D'Souza, H. D. Thames, and D. A. Kuban, "Dosevolume conundrum for response of prostate cancer to brachytherapy: summary dosimetric measures and their relationship to tumor control probability," International Journal of Radiation Oncology Biology Physics, vol. 58, no. 5, pp. 1540-1548, 2004.

[12] M. Zaider, "Permanent-implant brachytherapy in prostate cancer," in New Technologies in Radiation Oncology, pp. 379388, Springer, Berlin, Germany, 2006.

[13] S. Mallick, R. Azzouzi, L. Cormier, D. Peiffert, and PH. Mangin, "Urinary morbidity after I brachytherapy of the prostate," BJU International, vol. 92, no. 6, pp. 555-558, 2003.

[14] Z. A. Allen, G. S. Merrick, W. M. Butler et al., "Detailed urethral dosimetry in the evaluation of prostate brachytherapyrelated urinary morbidity," International Journal of Radiation Oncology Biology Physics, vol. 62, no. 4, pp. 981-987, 2005.

[15] M. Neill, G. Studer, L. Le et al., "The nature and extent of urinary morbidity in relation to prostate brachytherapy urethral dosimetry," Brachytherapy, vol. 6, no. 3, pp. 173-179, 2007.

[16] J. Crook, N. Fleshner, C. Roberts, and G. Pond, "Long-term urinary sequelae following iodine prostate brachytherapy," Journal of Urology, vol. 179, no. 1, pp. 141-146, 2008.

[17] T. Ohashi, A. Yorozu, K. Toya, S. Saito, and T. Momma, "Acute urinary morbidity following I-125 prostate brachytherapy," International Journal of Clinical Oncology, vol. 10, no. 4, pp. 262-268, 2005.

[18] S. A. Shah, R. R. Cima, E. Benoit, E. L. Breen, and R. Bleday, "Rectal complications after prostate brachytherapy," Diseases of the Colon and Rectum, vol. 47, no. 9, pp. 1487-1492, 2004.

[19] J. N. Shah and R. D. Ennis, "Rectal toxicity profile after transperineal interstitial permanent prostate brachytherapy: use of a comprehensive toxicity scoring system and identification of rectal dosimetric toxicity predictors," International Journal of Radiation Oncology Biology Physics, vol. 64, no. 3, pp. 817-824, 2006.

[20] F. M. Waterman and A. P. Dicker, "Probability of late rectal morbidity in I prostate brachytherapy," International Journal 
of Radiation Oncology Biology Physics, vol. 55, no. 2, pp. 342353, 2003.

[21] T. Ohashi, A. Yorozu, K. Toya et al., "Rectal morbidity following I-125 prostate brachytherapy in relation to dosimetry," Japanese Journal of Clinical Oncology, vol. 37, no. 2, pp. 121126, 2007.

[22] B. R. Prestidge, W. S. Bice, I. Jurkovic, E. Walker, S. Marianne, and A. Sadeghi, "Cesium-131 permanent prostate brachytherapy: an initial report," International Journal of Radiation Oncology, Biology, Physics, vol. 63, pp. S336-S337, 2005.

[23] V. E. Nuttens and S. Lucas, "AAPM TG-43U1 formalism adaptation and Monte Carlo dosimetry simulations of multipleradionuclide brachytherapy sources," Medical Physics, vol. 33, no. 4, pp. 1101-1107, 2006.

[24] V. E. Nuttens and S. Lucas, "Determination of the prescription dose for bi-radionuclide permanent prostate brachytherapy," Medical Physics, vol. 35, no. 12, pp. 5451-5462, 2008.

[25] R. G. Dale and B. Jones, "The clinical radiobiology of brachytherapy," British Journal of Radiology, vol. 71, pp. 465483, 1998.

[26] J. Z. Wang, N. A. Mayr, S. Nag et al., "Effect of edema, relative biological effectiveness, and dose heterogeneity on prostate brachytherapy," Medical Physics, vol. 33, no. 4, pp. 1025-1032, 2006.

[27] V. Antipas, R. G. Dale, and I. P. Coles, "A theoretical investigation into the role of tumour radiosensitivity, clonogen repopulation, tumour shrinkage and radionuclide $\mathrm{RBE}$ in permanent brachytherapy implants of I and Pd," Physics in Medicine and Biology, vol. 46, no. 10, pp. 2557-2569, 2001.

[28] M. J. Zelefsky, Y. Yamada, G. N. Cohen et al., "Five-year outcome of intraoperative conformal permanent I-125 interstitial implantation for patients with clinically localized prostate cancer," International Journal of Radiation Oncology Biology Physics, vol. 67, no. 1, pp. 65-70, 2007. 


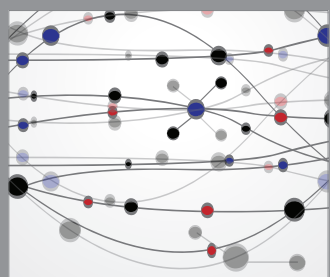

The Scientific World Journal
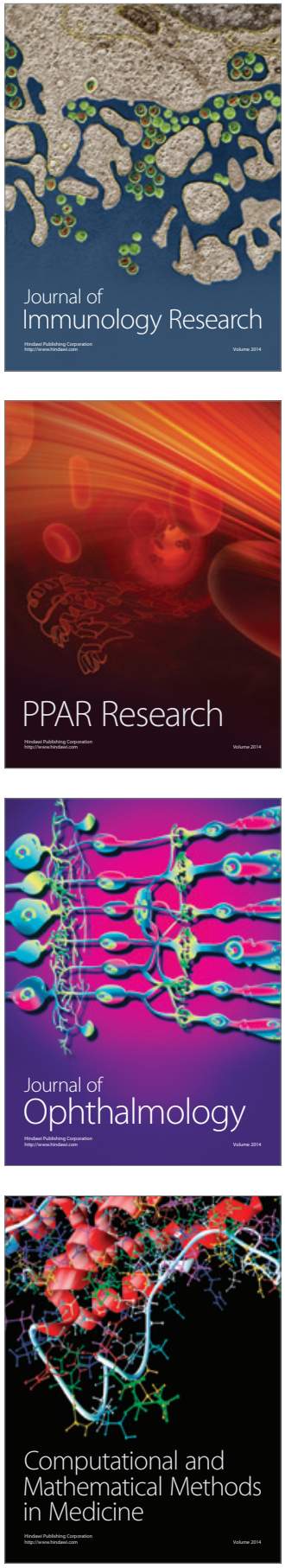

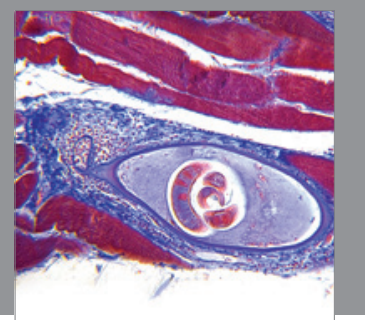

Gastroenterology

Research and Practice
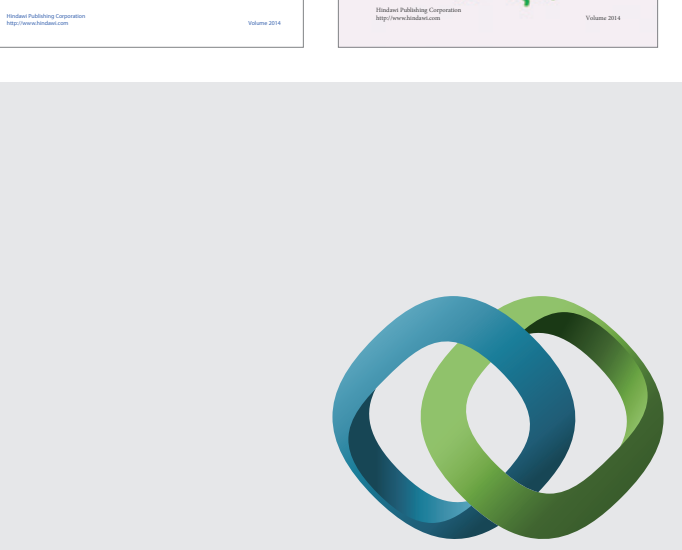

\section{Hindawi}

Submit your manuscripts at

http://www.hindawi.com
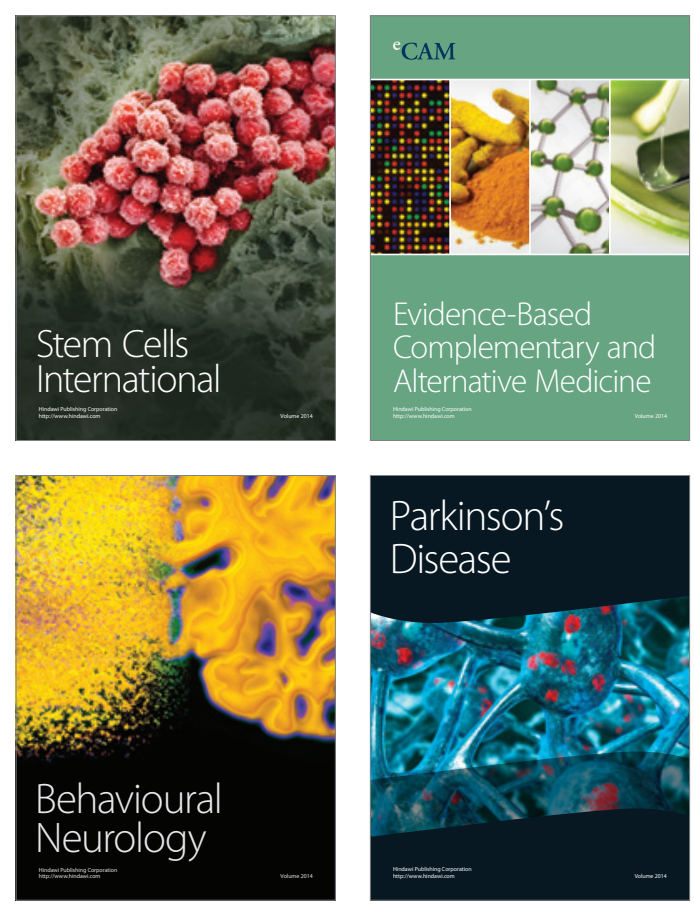

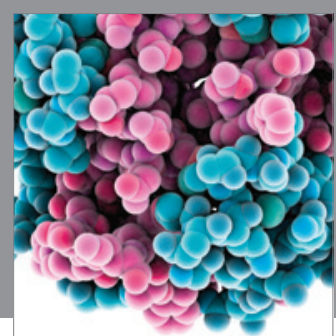

Journal of
Diabetes Research

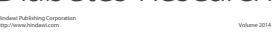

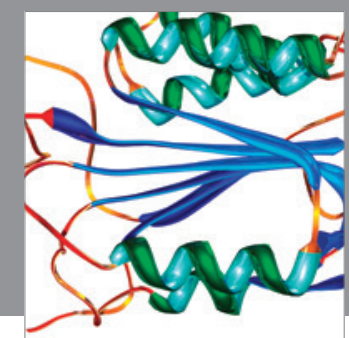

Disease Markers
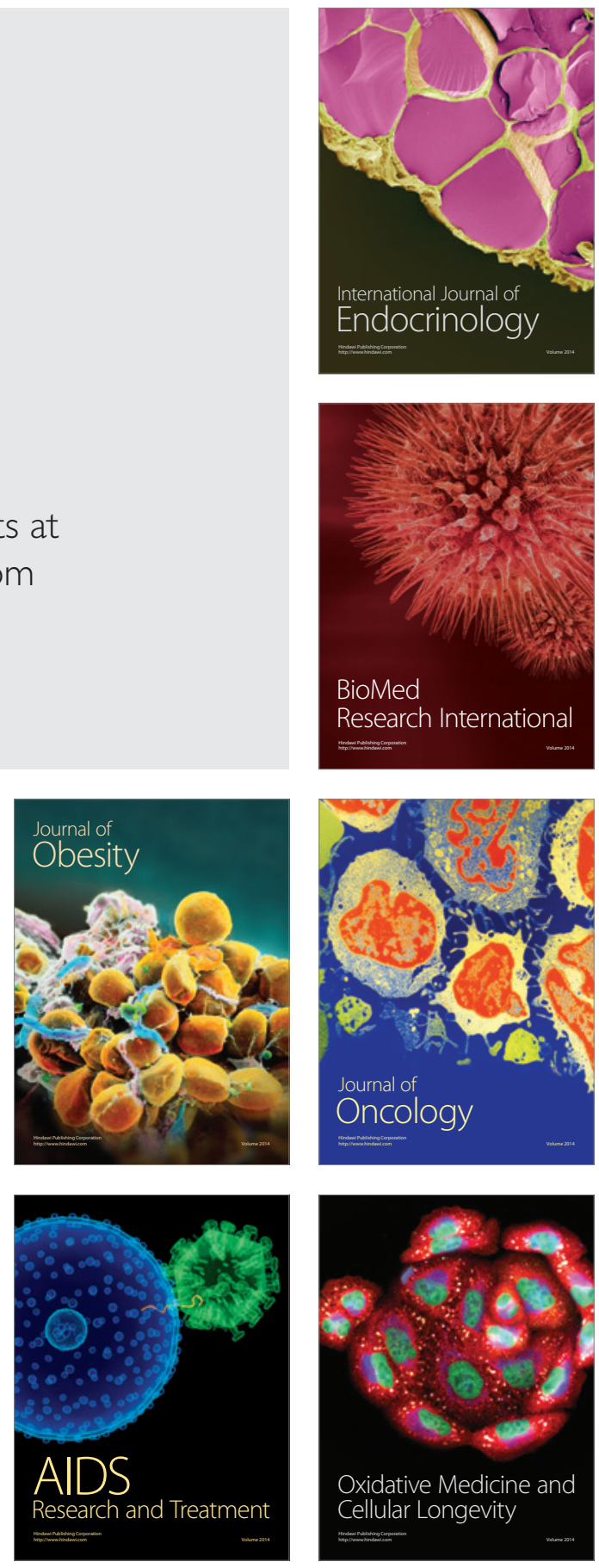